\title{
Relationship between spiritual intelligence and perceived stress among critical care nurses
}

\author{
A. Shahrokhi ${ }^{1}, \underline{\text { N. Elikaei }}^{2}$, L. Yekefallah ${ }^{1}$, A. Barikani $^{3}$ \\ ${ }^{1}$ Department of Critical Care Nursing, School of Nursing and Midwifery, Qazvin University of Medical Sciences, \\ Qazvin, Iran \\ ${ }^{2}$ School of Nursing and Midwifery, Qazvin University of Medical Sciences, Qazvin, Iran \\ ${ }^{3}$ Department of Social Medicine, Faculty of Medicine, Qazvin University of Medical Sciences, Qazvin, Iran \\ Corresponding Address: Nooshin Elikaei, School of Nursing and Midwifery, Qazvin University of Medical Sciences, \\ Qazvin, Iran. \\ Tel: +98-28-33338034; Email: nooshinelikaei@gmail.com \\ Received: 25 Dec 2017; Accepted: 16 May 2018
}

\section{Abstract}

Background: Spiritual intelligence as a basis of individual beliefs plays a fundamental role in various areas of human life, especially mental health promotion.

Objective: This study investigated the relationship between spiritual intelligence and perceived stress among critical care nurses.

Methods: This descriptive-analytical study was carried out on 115 nurses who were selected by nonrandom sampling method in a correlation manner. Data were gathered by using three questionnaires: demographic information, king's spiritual intelligence and Cohen's perceived stress questionnaire and analyzed by linear regression and correlation coefficient analysis.

Findings: In this study 13 men and 102 women participated. The mean score of perceived stress $(25.7 \pm 5.96)$ and spiritual intelligence score $(50.28 \pm 14.35)$ were moderate. There was a reverse and significant relationship between spiritual intelligence and its subscales with perceived stress $(\mathrm{r}=-0.243$ and $\mathrm{P}=0.009)$. The study showed that spiritual intelligence and education level can be considered as predictive variables for perceived stress $(\mathrm{P}<0.001)$.

Conclusion: Considering the significant relationship between spiritual intelligence and perceived stress and the predictive role of spiritual intelligence, one of the effective strategies in reducing stress can be shifting the attention of nurses towards spiritual intelligence and attempt to promote it.

Keywords: Spirituality, Intelligence, Spiritual intelligence, Critical care, Nurses, Perception Citation: Shahrokhi A, Elikaei N, Yekefallah L, Barikani A. Relationship between spiritual intelligence and perceived stress among critical care nurses. J Qazvin Univ Med Sci 2018; 22(3): 4049. 


\title{
ارتباط استرس ادراك شده و هوش معنوى در يرستاران بخشهاى مراقبت ويزه
}

\author{
اكرم شاهرخى'، نوشين اليكايي'، دكتر ليلى يكه فلاح'، دكتر آمنه باريكانى"
}

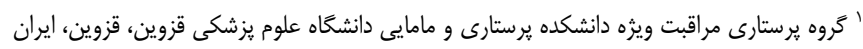

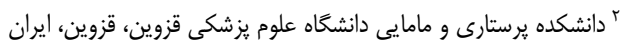

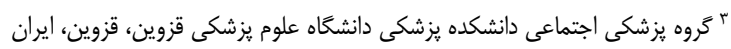

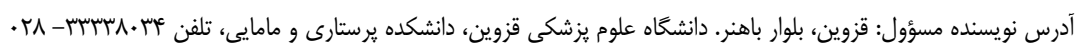

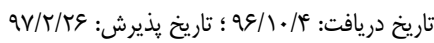

زمينه: هوش معنوى بهعنوان زير بناى باورهاى فرد، نقش اساسى در زمينهایى مختلف زنداكى انسان به ويزّه تأمين و ارتقاى سلامت روانى دارد.

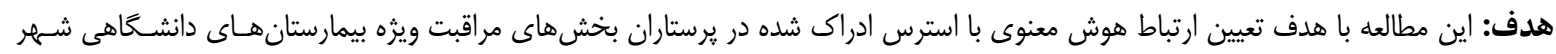
قزوين انجام شد.

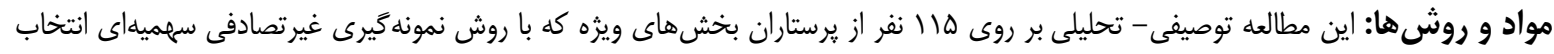

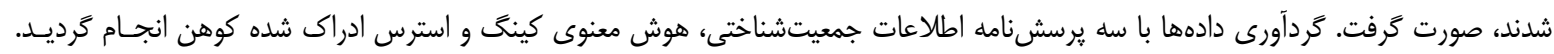

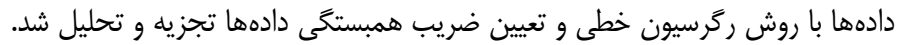

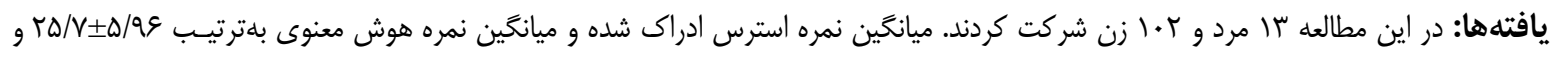

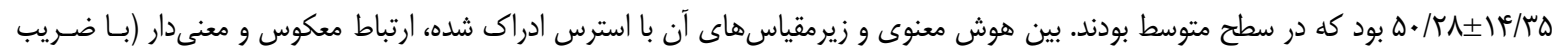

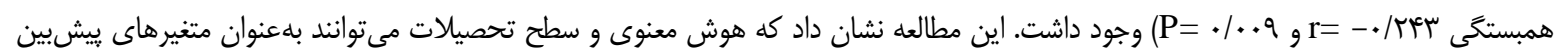

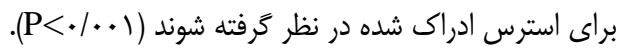

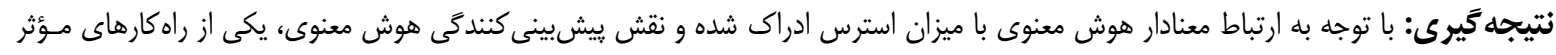

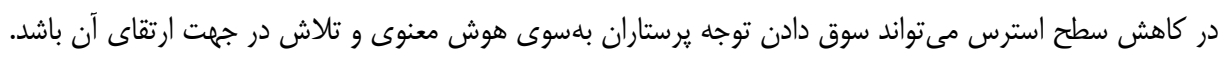

أسيبهـاى جراحسى، سـوختخى هـا، تحريكـات هيجـانى،

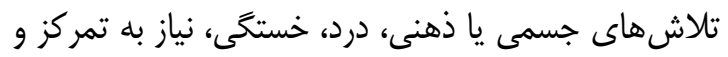

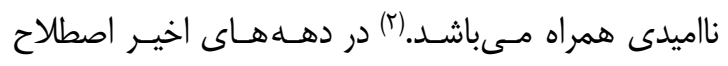
استرس به محركى اطلاق مىشود كه مىتواند تغييراتى را

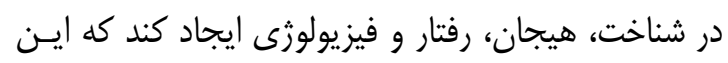

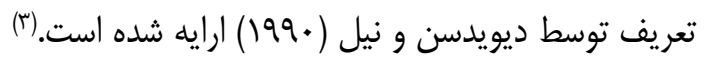
شغل يكى از مهمترين دلايل اين استرس در زندگى لونى

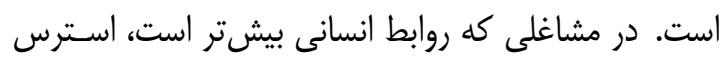
نيز بالاتر است(أ) در اين ميان بيمارستانها و ساير مراكـز

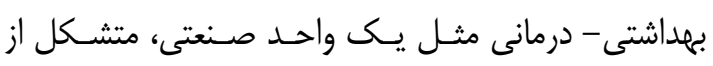
عوامل توليدى از قبيل؛ سرمايه، نيروى انسانى، تكنولوزى دئ دئى
استرس يـك واكـنش در مقابـل تهلديــد ادراك شــه

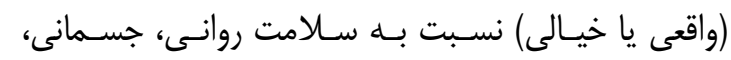

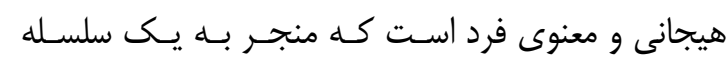
ياسخها و در نتيجه سازگًارىهاى فيزيولوزيـك مسى وشـود. استرس شديد يا طولانى مىتواند منجر به شروع، تشديد و

تداوم بسيارى از كسالتهاى روانى و جسمانى گردد. (1) تعريف سليه از استرس در مبحث سـلامت و بيمـارى

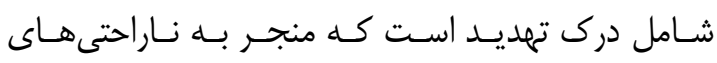
اضطرابى، تنش هاى عاطفى و مشكل در قضاوت مى گردد.

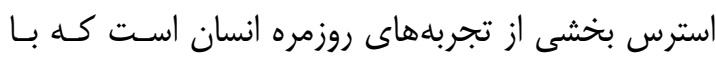

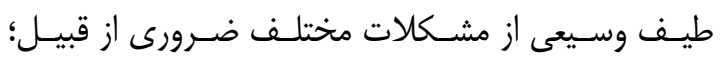


اساسى تبيين كننده احتمال اتخاذ راهبرد مقابله توسط افراد در موقعيتهاى استرسزا محسـوب مىشـود. به احتمـال

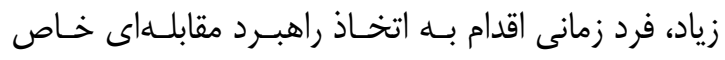

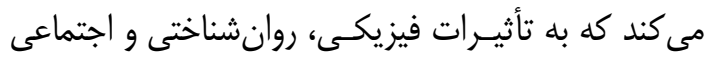

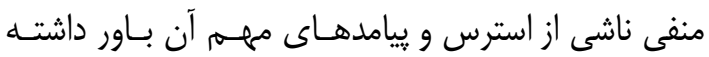

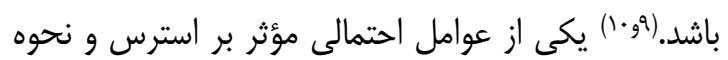

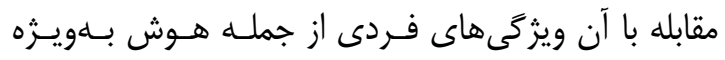

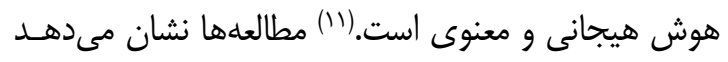

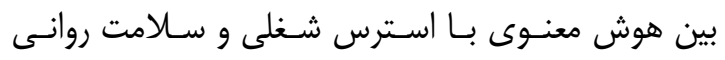

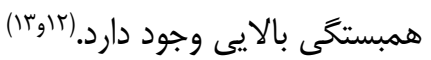
هوش معنوى بلهعنوان زيربنـاى باورهـاى فـرد نقـش داردي

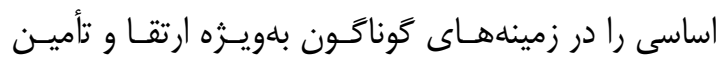

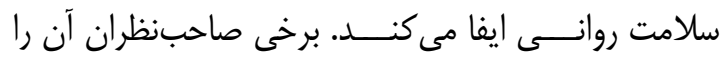
متضمن بالاترين سطوح زمينههاى رشد شناختى، اخلاقى،

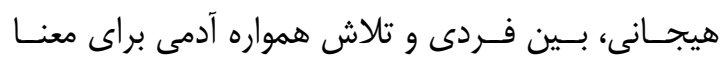

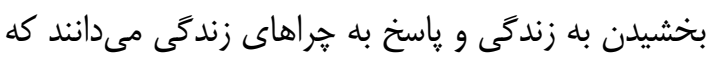

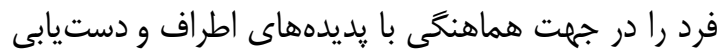

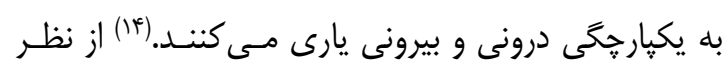

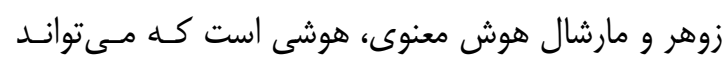

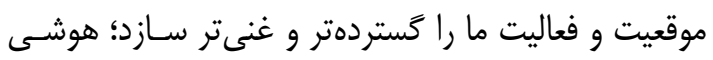

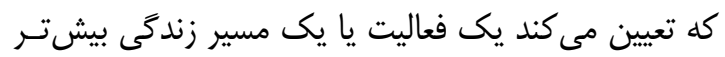

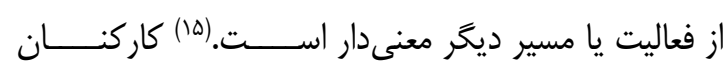

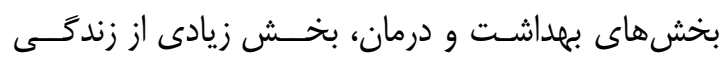

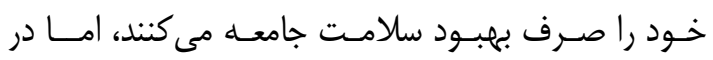

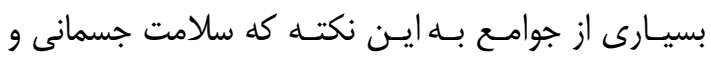
روانى خود آنها نيز ممكن است با درك مستمر درد و رونج بيماران به مخاطره بيفتد توجه نمى شود. (1)

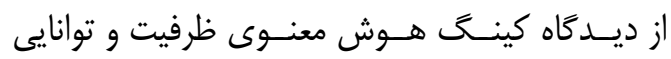
منحصر به فردى را در شخص ايجاد مى كند و داراى جهار

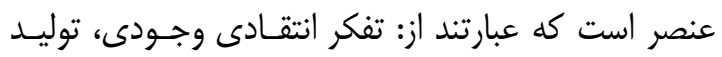

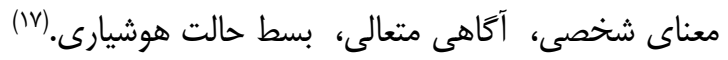

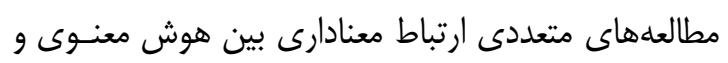
واكنشهاى هيجانى نظير؛ استرس، اضطراب و افسردگى،

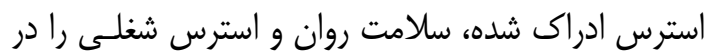

و مديريت مىباشند كه براى ايجاد بازدهى به نـام حفـظة

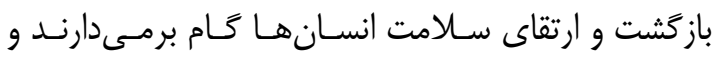

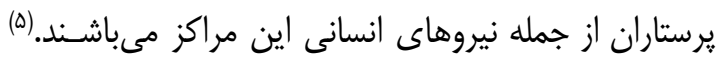

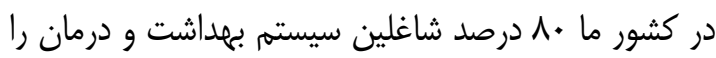

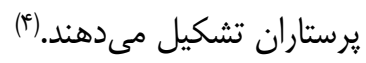
يرستارى مراقبتهاى ويزه يك شـاخه اختصاصسى از

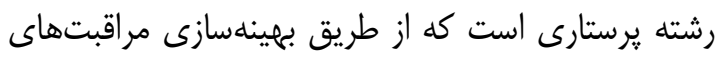

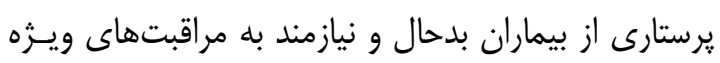

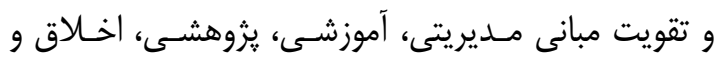

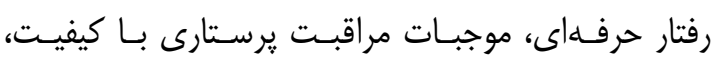

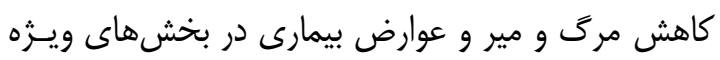

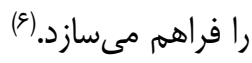
يرستاران بهعلت نياز به مهـارت و تمركـز بـالا، كـار

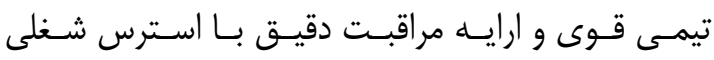
بيشترى مواجه هستند. مؤسسه امنيـت شـنلى آمريكـاى

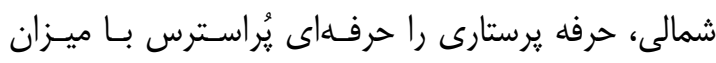

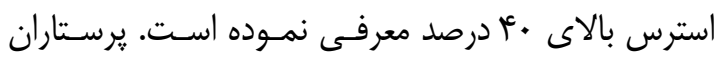

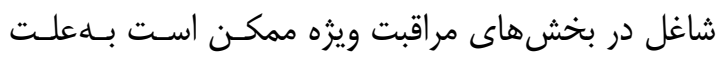

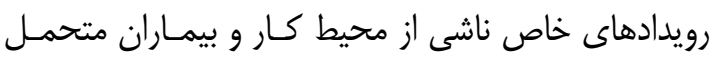

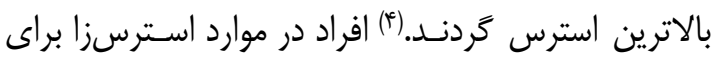

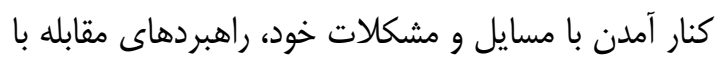

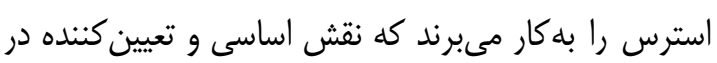

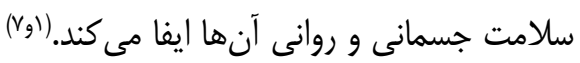

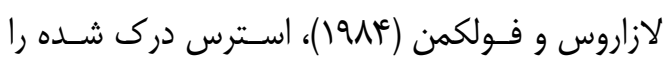

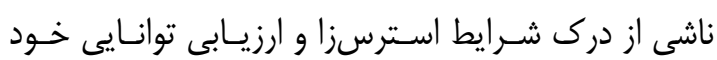

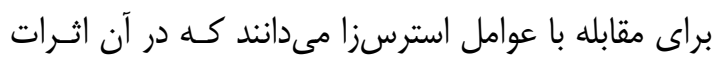

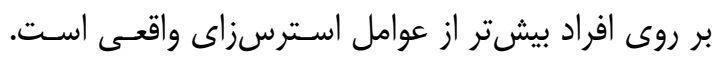

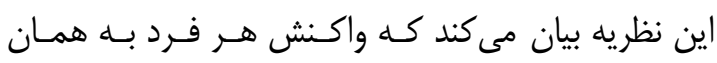

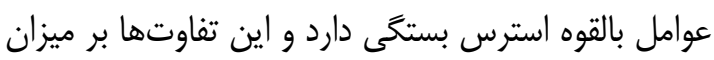
ارزيابى و توانايى حل مشكل تأثير مى كذارد.(1)

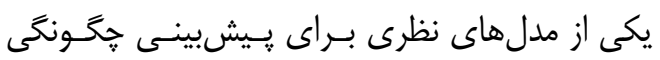

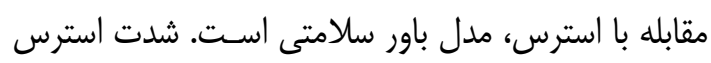

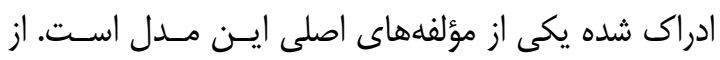
نظر اين مدل، شدت استرس ادراك شده يكى از مؤلفههاى 
معيارهاى ورود به مطالعه، نداشتن بيمارى روانشناختى

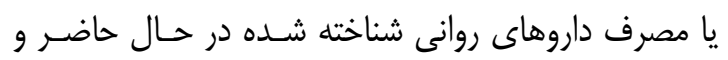

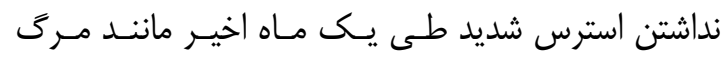

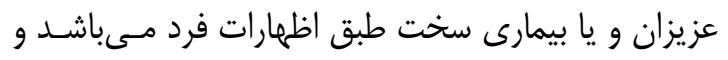

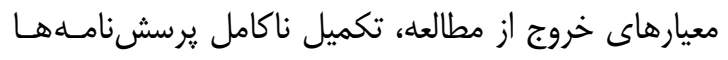

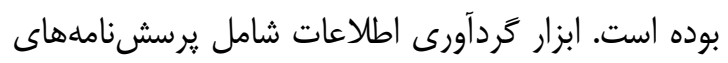

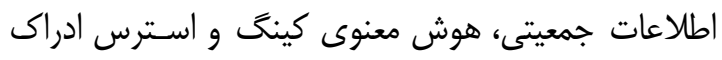
شده كوهن مىباشد.

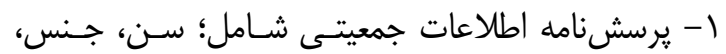
تأهل، سابقه كار، دانشعاه محل تحصيل، مدت زمات زمان كـار

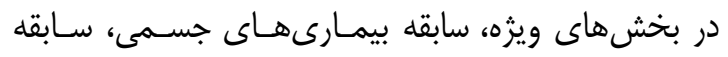

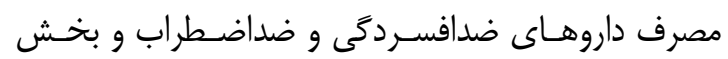
محل خدمت مىباشد.

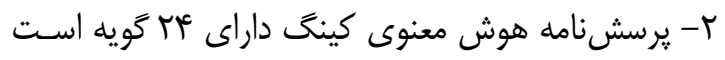

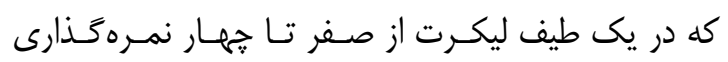

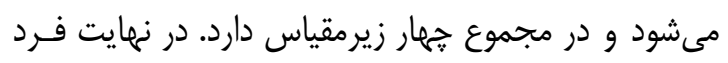

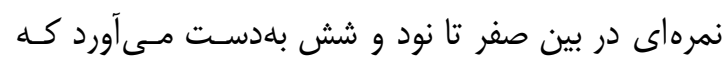

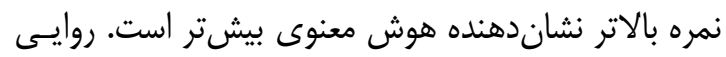

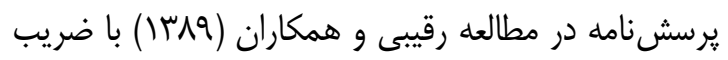

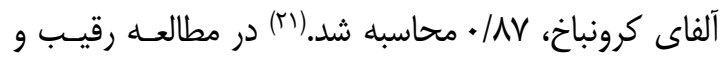

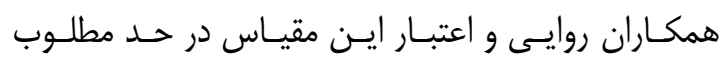

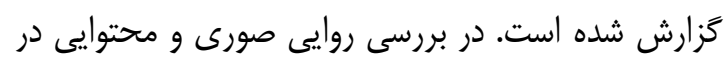

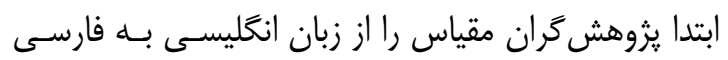

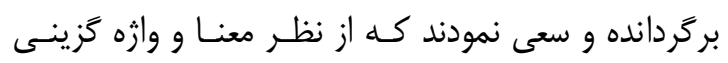

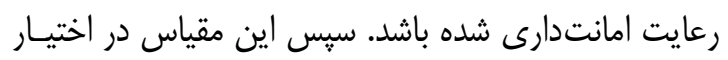

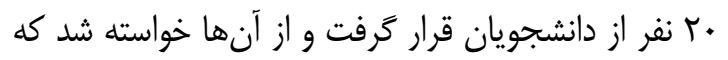

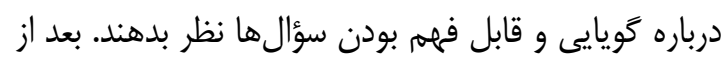

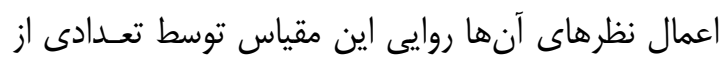

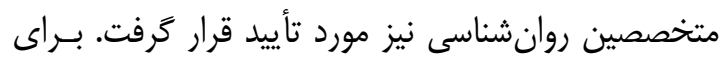

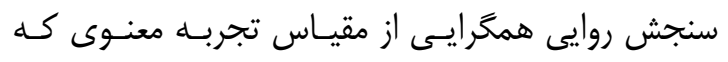

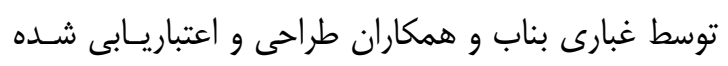

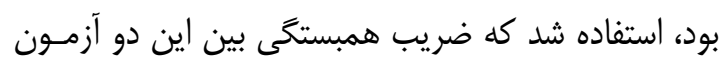

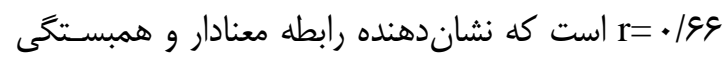
بالاى اين دو آزمون است.
افراد با مشاغل مختلف نشان مى دهند.(T/،هو 19) تعداد مطالعههاى كمى در زمينه استرس ادراك شده و

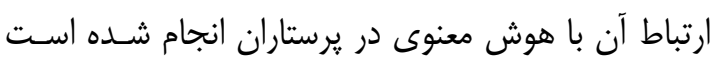

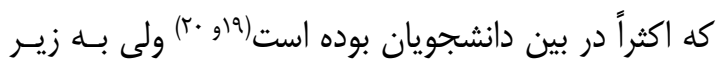

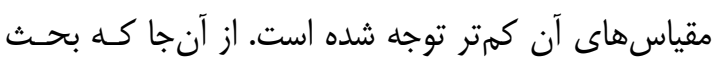

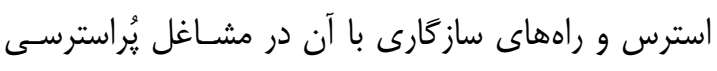

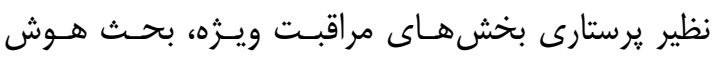

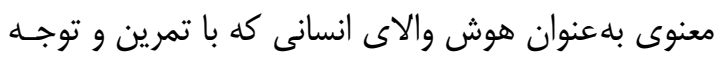

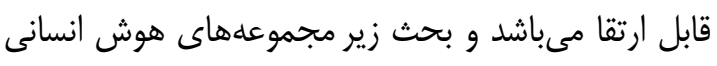
كه هر كدام بخش مهمى از معنويت را شـامل مسى شـونده،

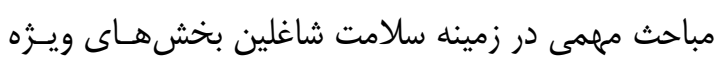

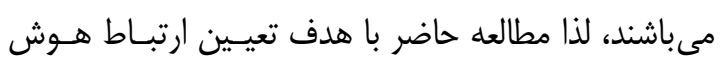

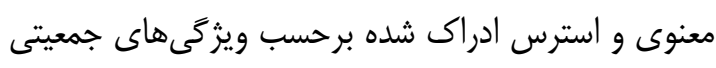

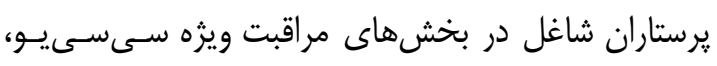

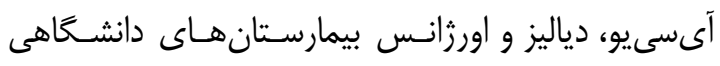
شهر قزوين طراحى و اجرا كرديد.

\section{مواد و روشها:}

مطالعـه از نـوع توصـيفى - تحليلـى (همبسـتخىى) در

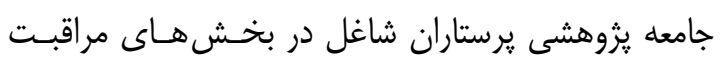

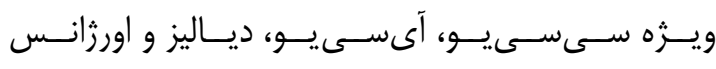

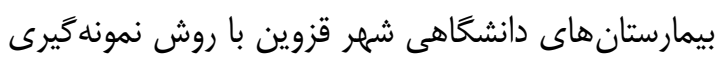

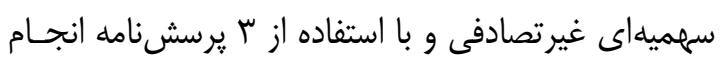

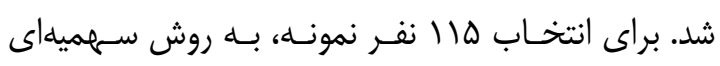

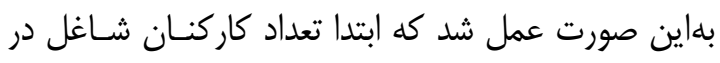

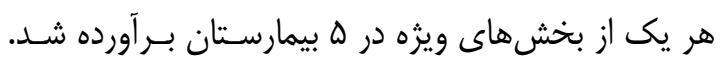

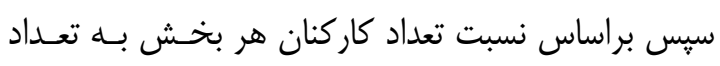

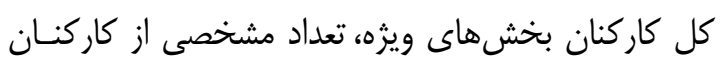

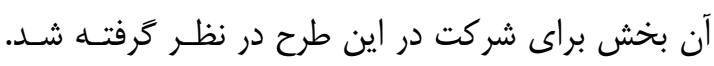

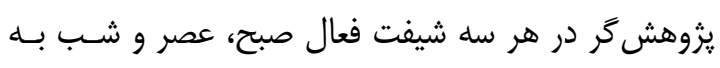

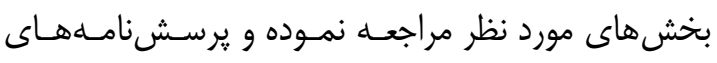

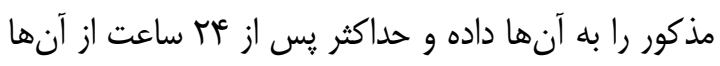

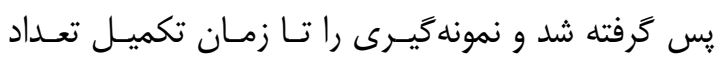
نمونهها به انجام رسانيد. 
تعداد ץ+1 نفر (19/9 درصد) مدرك كارشناسى קرسـتارى و rا نفر (ع/.1 درصد) كارشناسى ارشد و بـالاتر داشـتند.

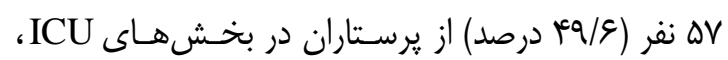

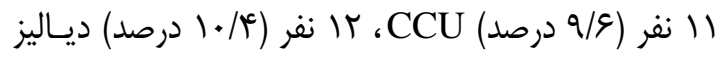

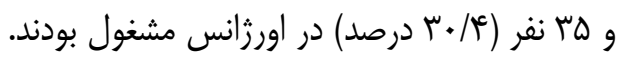

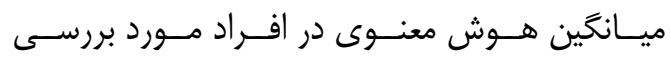
هَ/F

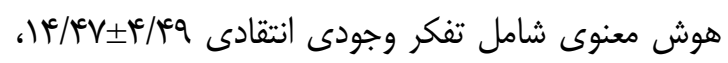

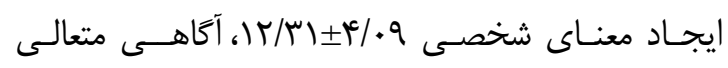

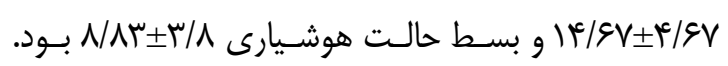

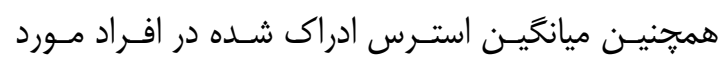

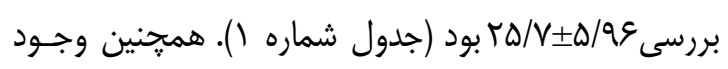

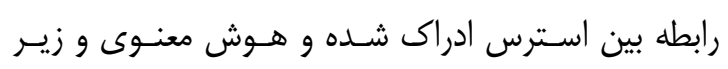

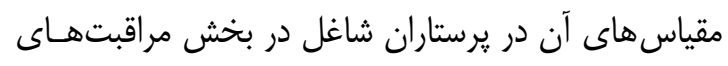

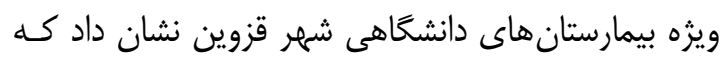

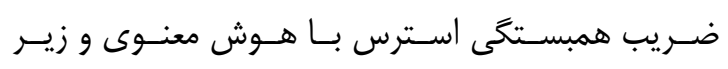

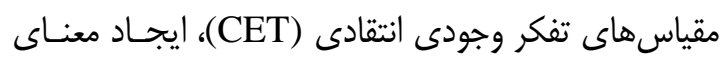

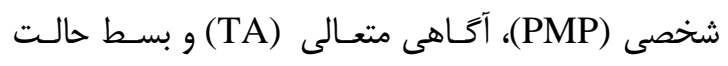

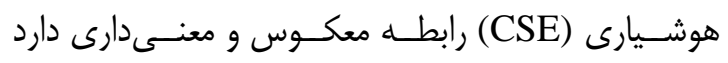
(جدول شماره r). با استفاده از آزمون رَرسيون خطى براى متغير وابسته

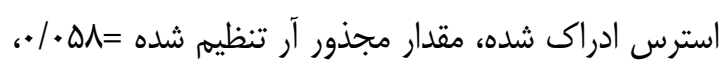

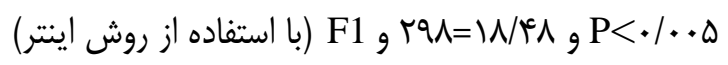

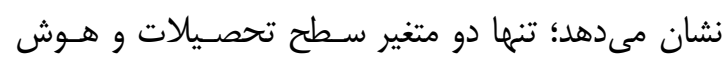

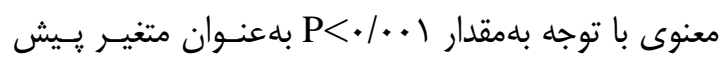

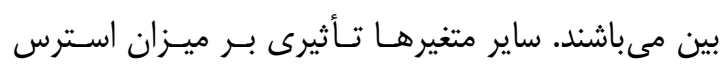
ادراك شده نداشتهاند (جدول شماره بار).
ץ- يرسـش نامــه اسـترس ادراك شـــه توسـط كـوهن و و

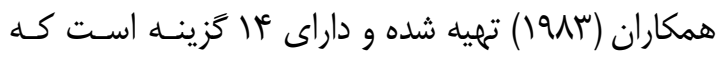

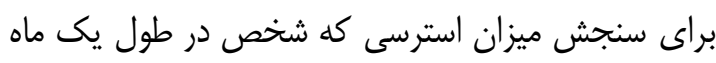

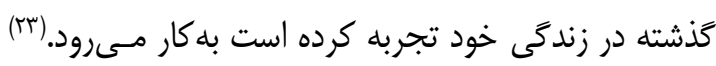

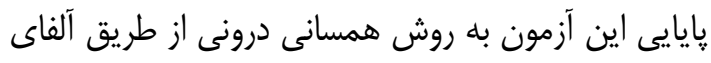

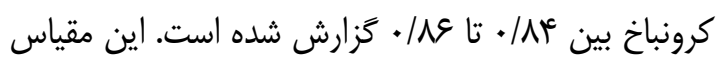

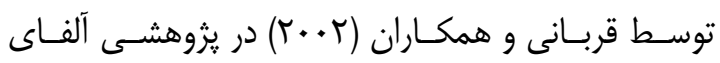

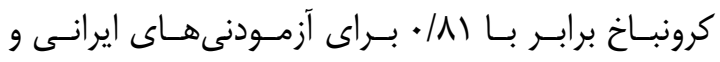

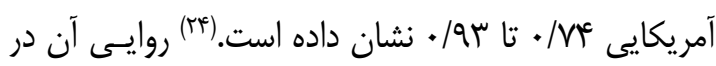

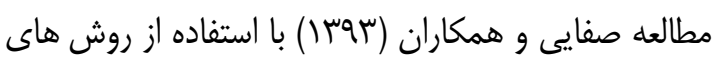

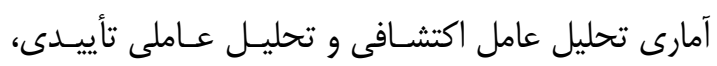

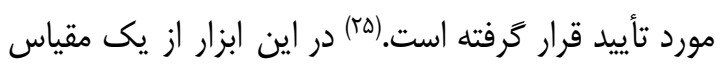
ينج زَينهاى هركز، خيلى كم، متوسط، نسبتاً زياد و خيلى

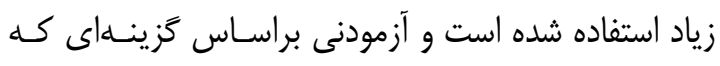

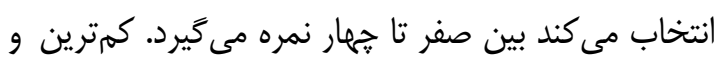

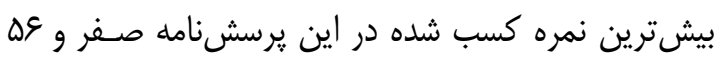

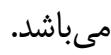

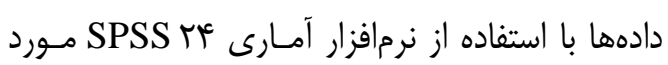

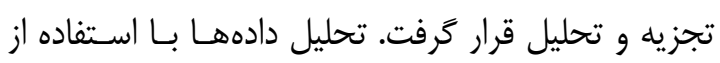

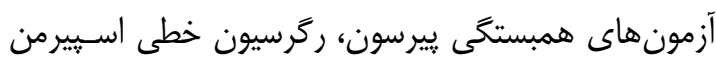

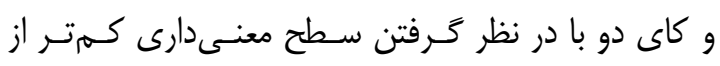
ه • • • محاسبه شد.

\section{(1) يافتها:}

از بين هال النفر يرستار مورد بررسى؛ سا با نفر (س/1/ درصد)

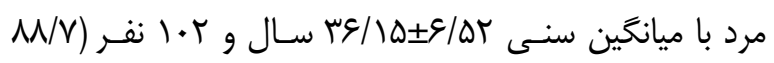

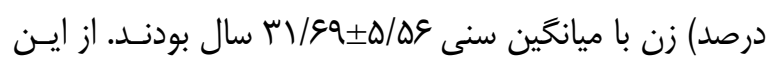

جدول ا - توزيع فراوانى يرستار ان بخشهاى مراقبت ويزه دانشًَاهى شهر قزوين برحسب ميانكَين هوش معنوىى زيرمقياس هاى هوش معنوى و استرس ادراك فئ شده

\begin{tabular}{|c|c|c|}
\hline دامنه تغييرات & ميانگينـ انحراف معيار & \\
\hline$q-\Lambda V$ & $\Delta \cdot / K \Lambda \pm \mid F / \widetilde{L} \Delta$ & هوش معنوى \\
\hline$\cdot-r \Lambda$ & $\mid f / F V \pm F /<q$ & تفكر وجودى انتقادى (CET) \\
\hline$\cdot-T^{\prime}$ & $|r / r| \pm F / \cdot q$ & ايجاد معناى شخصى (PMP) \\
\hline e & $\mid \Psi / \& V \pm F / \& V$ & آكاهى متعالى (TA) \\
\hline$\cdot-19$ & $\Lambda / \Lambda \mu \pm r / \Lambda$ & بسط حالت هوشيارى (CSE) \\
\hline $11-r \lambda$ & $r \Delta / V \pm \Phi / q \varepsilon$ & استرس ادراك شده \\
\hline
\end{tabular}


جدول Y- ميزان همبستكى (ييرسون) زيرمقياسهاى هوش معنوى و استرس در يرستاران

\begin{tabular}{|c|c|c|c|c|c|c|c|}
\hline ادراى شده & هوش هعنوى & بسط حالت & متعالى آكاهى & شخصى ايجاد معنى & تفكر وجودى & ضريب همبستكى ( ييرسون) & \\
\hline $\begin{array}{l}-. / 4 T A \\
\cdot / \cdot 1 f\end{array}$ & $\begin{array}{l}\cdot / 1<q \\
\cdot / \cdots\end{array}$ & $\begin{array}{l}. / p q . \\
. / \cdots\end{array}$ & $\begin{array}{l}. / 9 \wedge \mu \\
. / \cdots\end{array}$ & $\begin{array}{l}.|94| \\
.1 \ldots\end{array}$ & 1 & $\begin{array}{l}\mathrm{r} \\
\mathrm{p}\end{array}$ & تفكر وجودى انتقادى \\
\hline 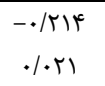 & $\begin{array}{l}\cdot / \Lambda F . \\
. / \cdots\end{array}$ & $\begin{array}{l}\cdot 10 \cdot r \\
.1 \cdot .\end{array}$ & $\begin{array}{l}\cdot / 9 \vee \wedge \\
. / \cdots\end{array}$ & 1 & $\begin{array}{l}.|94| \\
. / \ldots\end{array}$ & $\begin{array}{l}\mathrm{r} \\
\mathrm{p}\end{array}$ & ايجاد معناى شخصى \\
\hline $\begin{array}{l}-. / 1 F a \\
-. / 4 T\end{array}$ & $\begin{array}{l}. / 19 \vee \\
. / 1 \cdots\end{array}$ & $\begin{array}{l}. / 9 \mid 1 \\
.1 \%\end{array}$ & 1 & $\begin{array}{l}.19 \vee \wedge \\
.1 \ldots\end{array}$ & $\begin{array}{l}. / 9 \wedge \mu \\
. / \ldots\end{array}$ & $\begin{array}{l}\mathrm{r} \\
\mathrm{p}\end{array}$ & آََاهى متعالى \\
\hline $\begin{array}{l}-\cdot / 4 r q \\
. / \cdot\end{array}$ & $\begin{array}{l}. / \mathrm{V} \mu \\
. / \ldots\end{array}$ & 1 & $\begin{array}{l}. / 9 \mid 1 \\
. / \cdots\end{array}$ & $\begin{array}{l}.10 \cdot r \\
.1+.\end{array}$ & $\begin{array}{l}. / 4 q . \\
. / \ldots\end{array}$ & $\begin{array}{l}\mathrm{r} \\
\mathrm{p}\end{array}$ & بسط حالت هوشيارى \\
\hline $\begin{array}{l}-\cdot / \text { T } \\
. / \cdot 9\end{array}$ & 1 & $\begin{array}{l}. / V A r \\
. / \ldots\end{array}$ & $\begin{array}{l}. / 19 \vee \\
. / \cdots\end{array}$ & $\begin{array}{l}. / A r \\
. / \ldots\end{array}$ & $\begin{array}{l}. / 14 q \\
. / \ldots\end{array}$ & $\begin{array}{l}\mathrm{r} \\
\mathrm{p}\end{array}$ & هوش معنوى \\
\hline 1 & $\begin{array}{l}-\cdot / \text { TeT } \\
. / .99\end{array}$ & 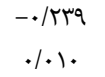 & $\begin{array}{l}-. / \text { IFD } \\
. / 4 T\end{array}$ & $\begin{array}{l}-/ \cdot k \mid \\
-\cdot / 4 \mid F\end{array}$ & $\begin{array}{l}-. / 4 t i k \\
-.4 k\end{array}$ & $\begin{array}{l}\mathrm{r} \\
\mathrm{p}\end{array}$ & استرس ادراك شده \\
\hline
\end{tabular}

جدول س- نتايج آزمون آناليز رتر سيون براى متغير هاى وابسته، مستقل و زمينهاى

\begin{tabular}{|c|c|c|c|c|c|}
\hline سطح معنىدارى & آماره آزمون & رَريب & انحراف معيار & ضريب رگر سيون & \\
\hline.$/ 110$ & . / RMK &.$/ \cdot \Delta T$ & תTKK & $.1 \cdot \Delta F$ & سن \\
\hline.$/ K \Lambda$ & $1 / \cdot 18$ & س & r/rqs & $r / F q f$ & جنس \\
\hline $.199 \mathrm{~V}$ & rTז/.- & $-\cdot / \cdot k^{c}$ & $1 / \cdot 9$ & $-\cdot / 4 V$ & تأهل \\
\hline.$/ \cdot r I^{*}$ & T/TQ & ./THE & I/AV & F/MAV & تحصيلات \\
\hline$\cdot / \Lambda \& Y$ &.$- / I V I$ & $-\cdot \cdot 1 \cdot 1 \mathrm{~V}$ & . / teq & $-\cdot \cdot \cdot v 9$ & بخش محل خدمت \\
\hline . lorq & $-\cdot|q \mu|$ & $-\cdot / 1 \cdot V$ & $\cdot / 4 \cdot r$ &.$- / 1 Y \Lambda$ & سابقه كار در بخش ويثه \\
\hline$\cdot|\wedge \varepsilon|$ &.$/ 1 V 9$ & $\cdot 1 \cdot+\mathrm{FV}$ & $\cdot / \mu \cdot r$ & 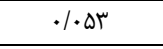 & سابقه كار يرستارى \\
\hline.$/ 091$ &.$- /$ ATA & $-\cdot \cdot \cdot \cdot n$ &.$|90|$ & . MFE & وضعيت استخدام \\
\hline.$/ 991$ & $-\cdot / \cdot+1$ & $.1 \cdot \cdot 1$ & $\cdot / \Delta \wedge \varepsilon$ & $.1 \cdot+1$ & 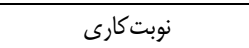 \\
\hline$\cdot 19 \cdot 0$ & $-\cdot / D T$ & $-\cdot / \cdot \Delta T$ & $r / 110$ & $-1 / .99$ & علاقه به رشته تحصيلى \\
\hline.$/ 1 \Delta S$ & I/FTA &.$/ 140$ & $1 / 019$ & $r / \Delta \& Q$ & علاقه به كار در بخش ويثه \\
\hline - ( ) & $-\cdot / 9 \cdot 0$ & $-\cdot / 1 \cdot r$ & $.1 \cdot 19$ & $-\cdot / \cdot 1)^{f}$ & ميزان اضافه كار \\
\hline (1./. & $-Y / / D S$ & $-\cdot / K I D$ & $\cdot|\cdot+4|$ &.$- .1 \cdot 19$ & هوش معنوى \\
\hline
\end{tabular}

* منى دار معنو

\section{بحث و نتيجه تيرى:}

ميزان هـوش معنـوى و جِهـار زيرمقيـاس آن (تفكـر

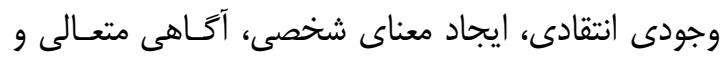

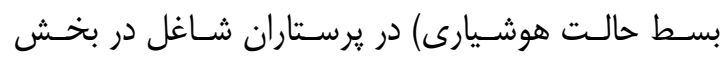

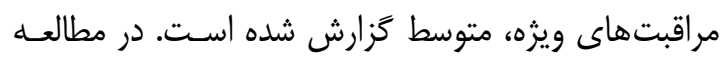

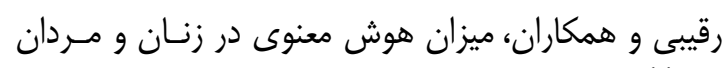

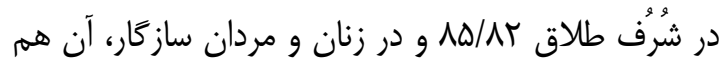

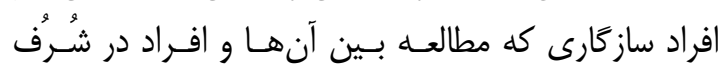

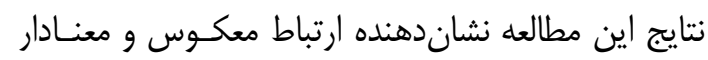
هوش معنوى و زيرمقياسهاى آن با استرس ادراك شــــه

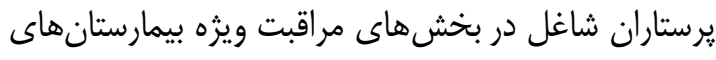

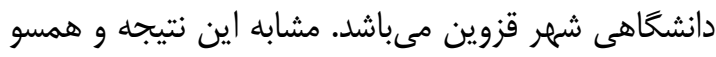

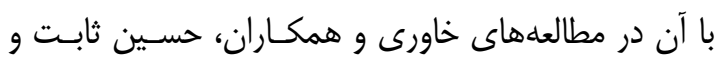

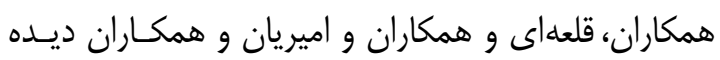

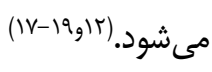




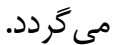

توجه بيشتر به مباحثى جهت تأمين و ارتقاى سلامت

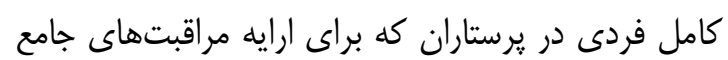

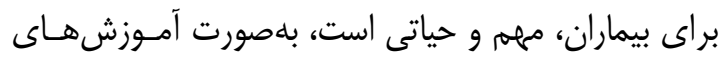

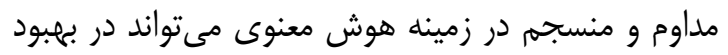
و بالا بردن آن نقش مؤثرى داشته باشد.

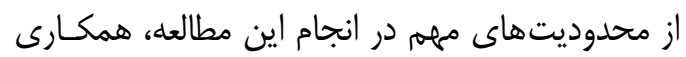

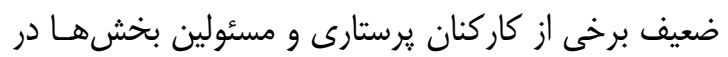

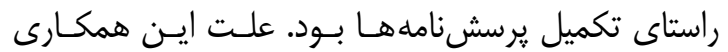

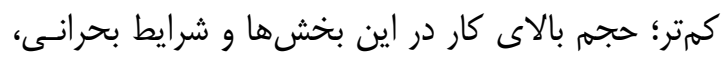

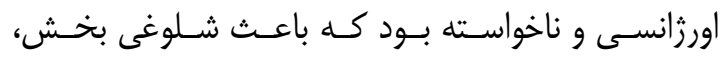
خستخى زياد كاركنان و كمبـود وقـت شـــ بــود. راهكـار

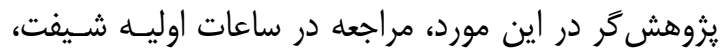

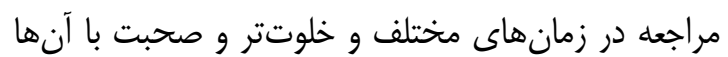

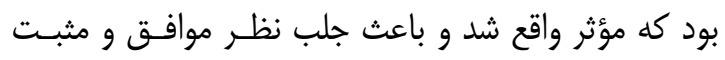
كاركنان براى همكارى بود.

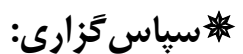

بدينوسيله از مسئولين محترم دانشـكده يرسـتارى و

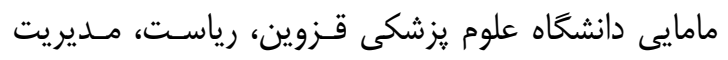

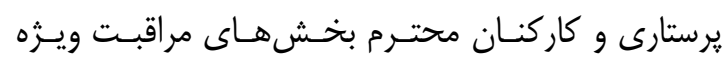
بيمارستانهاى دانشخاهى شهر قزوين شامل؛ بوعلى سينا،

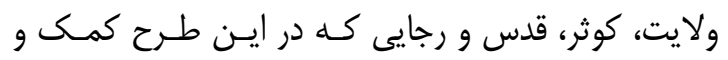
همكارى زيادى مبذول داشتند، كمال تشكر را داريم. ايسن

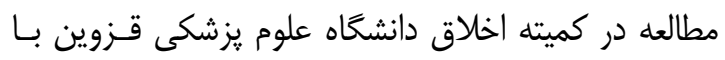

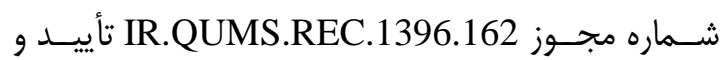

انجام يزوهش از لحاظ اخلاقى بلامانع بود.

\section{مراجع:}

1. Moghadam M, Rashidzadeh S, Shamsalizadeh N, Fallahi B. The impact of stress management training on the copping style and perceived stress in medical students. J Kurdistan Univ Med Sci 2014; 19(3): 52-60. [In Persian]
طلاق صورت كَرفته است، با يرستاران اين طرح مطابقـت

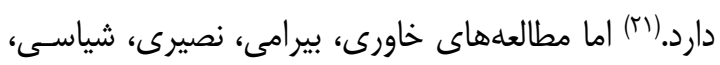

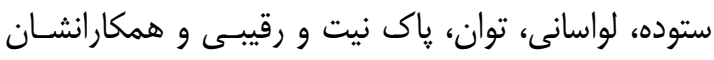

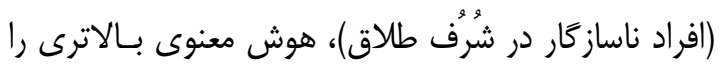

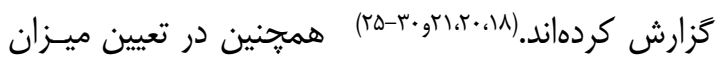

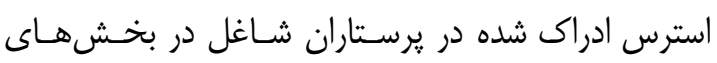

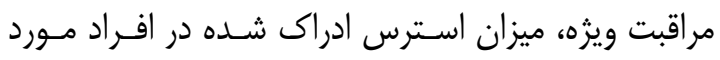

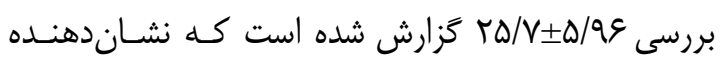
ميزان متوسط استرس در اين افراد بوده است. در مطالعها

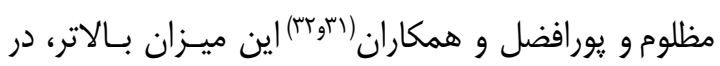

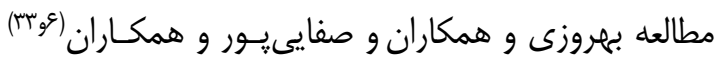

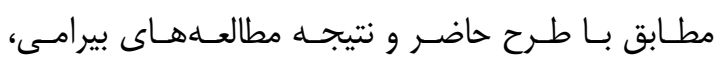

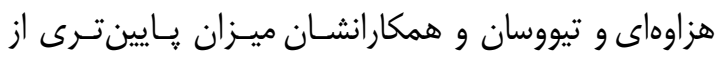

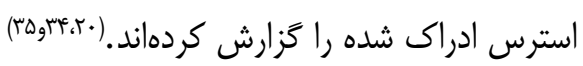

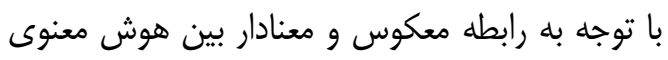

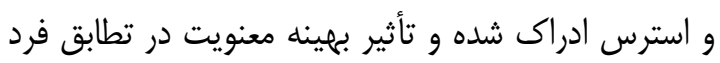

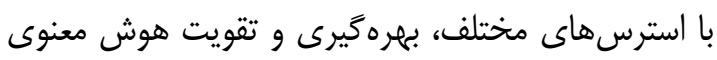

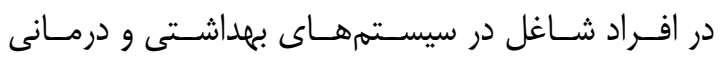
بهخصوص يرستاران كه با استرسهاى متعددى در محـل

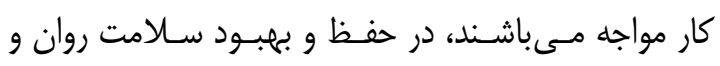

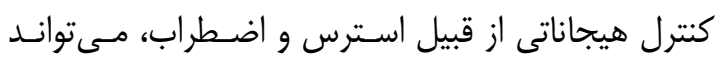

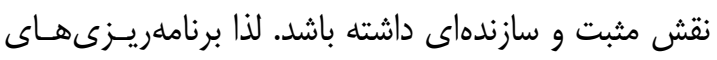

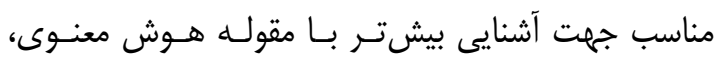

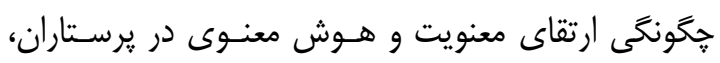

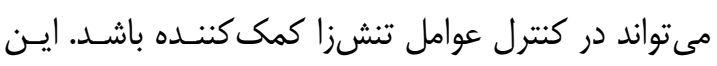

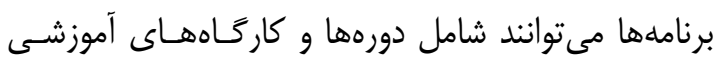

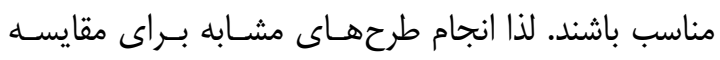

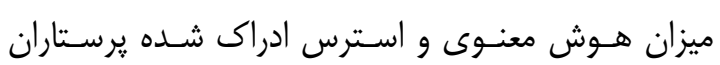

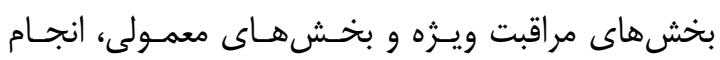

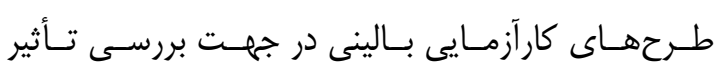

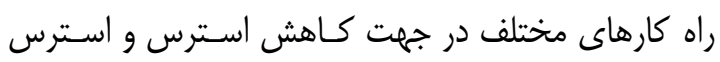

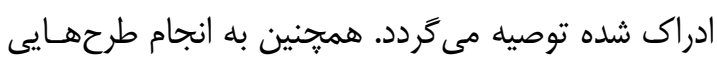

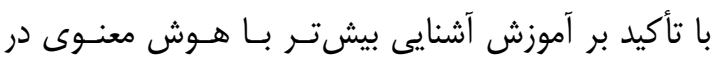

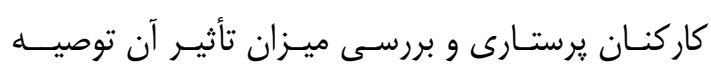


2. Fink, G. Stress: Concepts, Definition and History. Neuroscience and Biobehavioral Psychology, Elsevier, 2017. 1-9. doi: 10. 1016/B978-008045046-9.00076-0

3. KhodayariFard M, Parand A. Stress and ways to deal with it. 2nd ed Tehran: Tehran University Publishers; 2012: 1-2.

4. Latifzadeh S, Zarea K. Occupational stress and its related factors in nurses working in intensive care units of educational hospitals in Ahwaz, Iran. J Nov Appl Sci 2015; 4(4): 483-7.

5. Parvin N, Kazemian A, Hassan poor A, Alavi A. Evaluation nurses, job stressors in Shahrekord hospitals affiliated to medical university in 2002. Mod Care J 2005; 2 (3 and 4): 31-5. [In Persian]

6. Safaee Pour Zamanee M, Arefi M. The relation between coping styles and perceived stress in nurses of educational hospitals in Kermanshah University of medical science. J Clin Res Paramed Sci 2015; 4(1): 34-42. [In Persian]

7. Daneshpour A, Sarvghad S. The relation between metacognitive strategies with aggression and coping styles in Shiraz high school students. J Psychological Models and Methods 2011; 1(2): 25-43. [In Persian]

8. Abdollahi A, Abu Talib M, Yaacob SN, Ismail Z. Hardiness as a mediator between perceived stress and happiness in nurses. $\mathbf{J}$ Psychiatr Ment Health Nurs 2014; 21(9): 78996. doi: 10.1111/jpm.12142.

9. Masoudnia E. Impact of perceived vulnerability on student's coping strategies in stressful situations: an appraisal of health belief model. Iranian J Psychiatric Nursing 2015; 3(3): 63-75. [In Persian]

10. Hashemi T, Paymannia B. The relationship between (D) type of personality, and perceived stress with health behaviors in women with breast cancer. Iranian $\mathbf{J}$ Psychiatric Nursing 2014; 1(4): 36-44. [In Persian]

11. Baezzat F, Sharifzadeh H. Relationship between spiritual intelligence and emotional intelligence with job stress in university employees. Quarterly J Career Organization Counsel 2013; 4(13): 55-68. [In Persian]

12. Ghaleei A, Mohajeran B, Mahmoodzadeh M. The relationship among spiritual intelligence, mental health and job stress in nurses in Imam Khomeini hospital of Mahabad. Sci J Hamadan Nurs Midwifery Fac 2015; 23(4): 14-21. [In Persian]

13. Mahmoudirad G, Bagherian F. Effects of spiritual intelligence training on nurses' job stress. Quarterly J Nursing Management 2015; 4(1): 69-79. [In Persian]

14. Kaur D, Sambasivan M, Kumar N. Impact of emotional intelligence and spiritual intelligence on the caring behavior of nurses: a dimension-level exploratory study among public hospitals in Malaysia. Appl Nurs Res 2015; 28(4): 293-8. doi: 10.1016/j.apnr.2015. 01.006.

15. Keshavarzi S, Yousefi F. The relationship between emotional intelligence, spiritual intelligence and resilience. J Psychology 2012; 16(3): 299-318.

16. Rezaian A, Hadizadeh Moghadam A, Naeiji M J. Designing the model of patients' satisfaction in hospitals: Impact of medical staff's emotional intelligence, spiritual intelligence, and affectivity. J Hospital 2012; 10(3): 1-8.

17. Amirian ME, Fazilat-Pour M. Simple and multivariate relationships between spiritual intelligence with general health and happiness. J Relig Health 2016; 55(4): 127588. doi: 10.1007/s10943-015-0004-y.

18. Khavari k, Abbasi R, Afshar A, Talebi M. 
Determining the relationship between spiritual intelligence and emotional responses of nurses working in Tehran University hospitals. Social Welfare Quarterly 2014; 14(53): 165-77. [In Persian]

19. Hosseinsabet F, Ataei H. The relationship between spiritual intelligence and patience and perceived stress in undergraduate students. J Res on Relig Health 2016; 2(1): 26-33. [In Persian]

20. Bayrami M, Movahedi Y, Movahedi M. The role of spiritual intelligence in perceived stress, anxiety and depression of Lorestan Medical University students (Iran). J Babol Univ Med Sci 2014; 16(1): 56-62. [In Persian]

21. Raghibi M, Gharehchahi M. An investigation of the relation between emotional and spiritual intelligence among well-adjusted and discordant couples. Sociology of Women 2013; 4(1): 123-40. [In Persian]

22. Raghib MS, Siadat SA, Hakimi nia B, Ahmadi SJ. King's Spiritual Intelligence Scale (SISRI-24) credit validation in students at Isfahan University. Scientific J Management System 2011; 17(1): 141-64.

23. Moghadam M, Rashidzadeh S, Shamsalizadeh N, Fallahi B. The impact of stress management training on the copping style and perceived stress in medical students. Scientific J kurdistan Univ Med Sci 2014; 19(3): 52-60. [In Persian]

24. Rahimi K, Hosseinsabet F, Sohrabi F. Effectiveness of Stress Management and Resiliency Training (SMART) on Perceived Stress and Attitude toward Drug Use among Addicted Men Prisoners. J Clinical Psychol Studies 2016; 6(22): 1-19. [In Persian]

25. Nasiri F, Ardalan M, Mirzakhani E. Relationship between Spiritual Intelligence and Communication Skills of Lorestan University Staffs. Organizational Culture Management 2015; 13(3): 667-89.

26. Shiasi m, Etebarian A, Zarrin abadi Z, Ahmadi M. The effect of spiritual intelligence on the productivity of employees at Isfahan University of Medical Sciences based on structural equation model. J Health Adm 2016; 19(63): 34-44. [In Persian]

27. Sotoodeh H, Shakerinia I, Kheyrati M, Dargahi S, Ghasemi Jobaneh R. Surveying the relationship between spiritual and moral intelligence and the psychological well-being of nurses. Iran J Med Ethics Hist Med 2016; 9(1): 63-73. [In Persian]

28. Gholamali Lavassani M, Afzali L, Davoodi M, Afkari shahrestan Z. Nurses' job burnout: the role of emotional intelligence, spiritual intelligence and hardiness. J Applied Psychol Res Quarterly 2016, 7(2): 1-15. [In Persian]

29. Tavan H, Tavan S, Ahmadi Z, Zandnia F. The relationship between intellectual intelligence and emotional intelligence and some demographic variables among students of the faculty of nursing and midwifery, Ilam University of Medical Sciences in 2014. Jorjani Biomed J 2015; 3(1): 127-34. [In Persian]

30. Pakniyat M, Javidi HA. Mediating role of citizenship behaviors for spiritual intelligent and organizational mental health. J Psychol Models Methods 2016; 7(23): 19-37. [In Persian]

31. Mazlom SR, Darban F, Vaghei S, Modarres GM, M KL, M S. The effect of Stress Inoculation Program (SIP) on nurses' Perceived stress in psychiatric ward. Evidence Based Care J 2012; 2(1): 35-44. doi: 10.22038/EBCJ.2012.388.

32. Pourafzal F, Seyedfatemi N, Inanloo M, 
Haghani H. Relationship between perceived stress with resilience among undergraduate nursing students. Hayat 2013; 19(1): 41-52. [In Persian]

33. Behroozi N, Shahni Yeilagh M, Pour Seyed S. Relationship between Perfectionism, Perceived Stress and Social Support with Academic Burnout. J Management System 2013; 5(20): 83-102. [In Persian]

34. Hazavehei MM, Hosseini Z, Moeini B,
Moghimbeigi A, Hamidi Y. Assessing stress level and stress management among Hamadan hospital nurses based on PRECEDE model. Horizon Med Sci 2012; 18(2): 78-85. [In Persian] 35. Tuvesson H, Eklund M. Psychosocial work environment, stress factors and individual characteristics among nursing staff in psychiatric in-patient care. Int $\mathbf{J}$ Environ Res Public Health 2014; 11(1): 1161-75. 